\title{
Clinical Evaluation of a Respiratory Gated Guidance System for Liver Punctures
}

\author{
S.A. Nicolau ${ }^{1}$, X. Pennec ${ }^{2}$, L. Soler ${ }^{1}$, and N. Ayache ${ }^{2}$ \\ ${ }^{1}$ IRCAD-Hopital Civil, Virtual-surg, 1 Place de l'Hopital, 67091 Strasbourg Cedex \\ \{stephane.nicolau, luc.soler\}@ircad.u-strasbg.fr \\ 2 INRIA Sophia, Epidaure, 2004 Rte des Lucioles, F-06902 Sophia-Antipolis Cedex
}

\begin{abstract}
We have previously proposed a computer guidance system for liver punctures designed for intubated (free breathing) patients. The lack of accuracy reported $(1 \mathrm{~cm})$ was mostly due to the breathing motion that was not taken into account. In this paper we modify our system to synchronise the guidance information on the expiratory phases of the patient and present an evaluation on 6 patients of our respiratory gated system.

Firstly, we show how a specific choice of patient allows us to rigorously and passively evaluate the system accuracy. Secondly, we demonstrate that our system can provide a guidance information with an error below $5 \mathrm{~mm}$ during expiratory phases.
\end{abstract}

\section{Introduction}

CT/IRM guided liver puncture is a difficult gesture which can dramatically benefit from a computer guidance system [31410 5]. Indeed, such systems can reduce the repetitive $\mathrm{CT} / \mathrm{MRI}$ images needed for needle adjustment and the reinsertion attempts that lengthen the intervention duration and increase radiation exposure (when CT-guided). Moreover, it can improve the insertion accuracy that currently depends on the practitioner's experience.

In a previous work 9], we have introduced in the operating room a guiding system for radio-frequency thermal ablation (RFA) and showed that this system meets the sterility and cumbersomeness requirements. Then, the system accuracy was evaluated on patients with a passive protocol neglecting the breathing influence. The accuracy results around $1 \mathrm{~cm}$ were much larger that those obtained on a phantom $(2 \mathrm{~mm})$ [ (Wacker et. al. obtained an equivalent result on a freely breathing pig [14). Indeed, liver displacement reaches $1 \mathrm{~cm}$ during shallow breathing [116.

A recent report shows that RFA ablation has to be performed on tumors which diameter is between 1 and $3 \mathrm{~cm}$ [11. Thus, our radiologists consider that a guidance system has to provide an accuracy above $5 \mathrm{~mm}$ to avoid destroying too much healthy cells when the needle tip is not perfectly centered in the tumor.Consequently, to provide useful guidance information to the practitioner, we cannot neglect the breathing deformations. Several approaches are possible to take the breathing into account. Firstly, we can use the predictive model of

N. Ayache, S. Ourselin, A. Maeder (Eds.): MICCAI 2007, Part II, LNCS 4792, pp. 77-85, 2007.

(C) Springer-Verlag Berlin Heidelberg 2007 
organ positions with respect to the breathing proposed by [4. Unfortunately, it is not accurate enough for our application (error of prediction above $5 \mathrm{~mm}$ for the liver). Secondly we can synchronize the guidance system on a particular point of the breathing cycle i.e. the preoperative image and the guidance information are respectively acquired and provided at the same point of the respiratory cycle. This approach is motivated by several studies that evaluate the repositioning error of the liver between 1 and $2 \mathrm{~mm}$ 1|16/2 12 15. Therefore, the cumulated error of the system components $(\simeq 3 \mathrm{~mm})$ and the repositioning error $(\simeq 2 \mathrm{~mm})$ should remain below $5 \mathrm{~mm}$.

This reasonable assumption has still not been demonstrated neither on animals nor patients: validation has been only performed on cadavers [3] or on living pigs without taking breathing into account [14. In this paper, we report an in vivo accuracy evaluation of our system with a respiratory gating technique. After a presentation of the system principles, we explain how the choice of specific patients allows us to develop a riskless protocol to evaluate rigorously the system accuracy. Finally, we present the experimental results obtained on 6 patients and demonstrate that the system accuracy fits the clinical requirements when the guidance information is provided during expiratory phases.

\section{System Components}

In our setup, two jointly calibrated cameras are viewing the patient lying on the CT-table who is under general anesthesia and ventilated $(70 \%$ of RFA are performed under general anesthesia in our local hospital). Radio-opaque markers with a ring shape are stuck on his abdominal skin and a black dot is printed inside each marker. Then, a preoperative CT acquisition is performed during an expiratory phase, the markers are removed and a 3D model of the patient (including his skin, liver, tumors and markers) is automatically obtained from the CT image (cf. top left Fig. 11) [13]. Then, this patient model is rigidly registered in the camera frame using radio-opaque markers, their position being extracted in both $\mathrm{CT}$ and video images. The marker extraction and matching is performed automatically and the registration is performed by minimisation of the Extended Projective Point Criterion (EPPC) (algorithmic details are further explained and validated in [7/6]). The needle position is also tracked in real-time by the cameras so that we can display on a screen its relative position with respect to the patient model to guide the practitioner (cf. right Fig. 1). The guidance information is provided only during the expiratory phases. These phases are automatically detected by the system using the reprojection error of CT markers in the video images. Indeed, this error computed in real-time is roughly sinusoidal and minimal during the expiration.

We remind here the four error sources when the system is used by the practitioner. Three of them are due to the system only: needle tracking, patient registration and organ repositioning. The last error source is the ability of the practitioner to follow the guiding information provided by the system (we call it guidance error). 

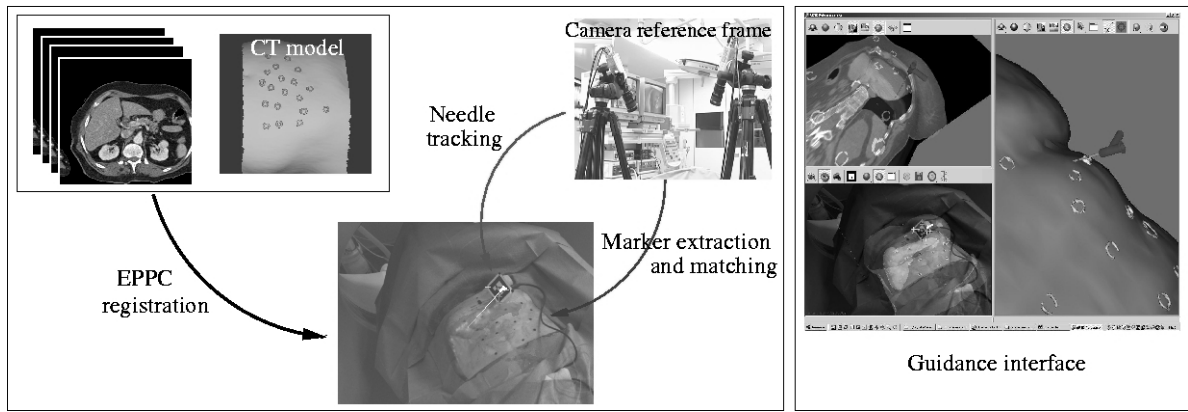

Fig. 1. Illustration of the system principles

\section{$3 \quad$ A Safe Evaluation Protocol with Specific Patients}

Hepatic tumors sometimes need contrast agent to be injected in the patient to be visible in the CT modality. For these patients, the clinical protocol to target tumors in interventional CT is slightly different from the standard one. A preoperative $\mathrm{CT}$ acquisition of the abdomen is realized with contrast agent. To guide the needle, the practitioner performs a mental registration of interventional CT slices with the preoperative CT image (in which tumors are visible). When he thinks the needle is correctly positioned, a second CT acquisition with contrast agent of the patient abdomen is performed. This second CT acquisition allows the practitioner to check the needle position with respect to the tumor he targeted.

The additional images available for these patients allow us to perform a passive evaluation of our system using the following data acquisition protocol:

\section{Experimental protocol}

Firstly, we stick homogeneously radio-opaque markers on the patient abdomen and a black dot is printed inside them. Then, a preoperative acquisition CT1 is realized in full expiration (it includes all the markers and the liver). The practitioner removes the markers and attaches to the needle a sterile pattern that allows its tracking. Then, he inserts the needle until he thinks he has correctly targeted the tumor. After the needle positioning, a stereoscopic video of the patient abdomen and needle is done during several breathing cycles. Finally, a second CT acquisition CT2 is done in full expiration, the needle remaining inside the patient (CT2 also includes the whole liver). This protocol does not change the information used by the practitioner to guide the needle: he realizes the intervention with his usual means (CT-slices) without any advice nor instruction from our system.

From the acquired experimental data, we can not only evaluate the system accuracy but also check that the needle remains straight during the insertion and that the repositioning error of abdominal structures at expiratory phases is negligible. To perform these three studies, we realize the three following evaluation processes:

\section{Evaluation of the liver repositioning error (cf. Fig. 2)}

We extract the spine, liver and skin in both CT1 and CT2. Then, the spine from $\mathrm{CT} 2$ is rigidly registered on the spine in $\mathrm{CT} 1$ with the Iterative Closest Point 
algorithm and the computed transformation is applied to liver and skin from CT2. This registration allows us to compare the relative movement of liver and skin with respect to a common rigid structure. To quantify these movements, we compute the distance between the liver (resp. skin) surface in CT1 with the liver (resp. skin) surface extracted from CT2 and registered in CT1.
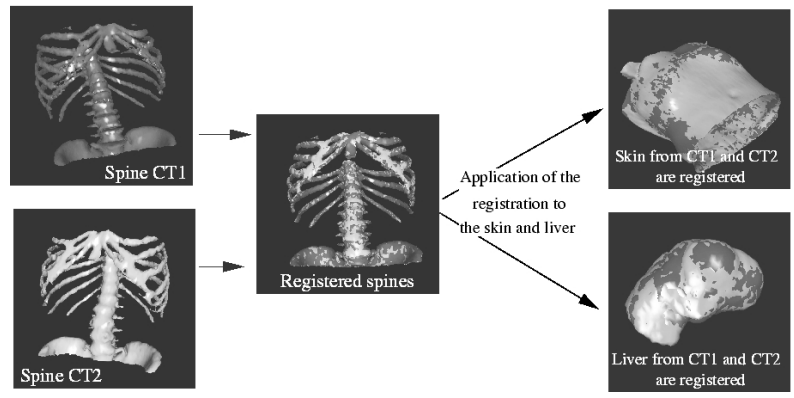

Fig. 2. To evaluate the repositioning error of liver and skin we firstly register the spines from CT1 and CT2. Then we apply the found rigid transformation to liver and skin surfaces and measure the distance between both surfaces.

\section{Evaluation of the needle curvature (Fig. 3)}

The needle in CT2 is extracted and we estimate orientations of the first and second half of its length. Then, we compare both orientations using the angular deviation $\alpha$ and the needle deflection.

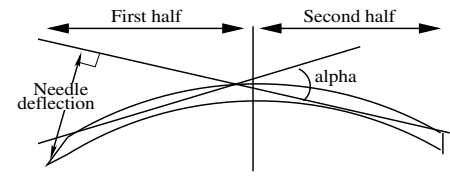

Fig. 3. Evaluation of the needle curvature

\section{Evaluation of the system accuracy (cf. Fig. 4)}

Liver and needle surfaces are extracted from CT2. The liver surface in CT2 is rigidly registered (using $\mathrm{ICP}$ ) on the liver surface in $\mathrm{CT} 1$ and the computed transformation is applied to the needle extracted in CT2. This registration provides the final needle position in the CT1 image. Then, we register the patient model from CT1 (with the needle) in the camera reference frame using the video image of the patient at full expiration. Finally, we evaluate the euclidean distance between the needle tip tracked by the camera (at expiration phase) and the needle tip in CT1 registered in the camera frame.

We call this distance system accuracy and emphasize it is an evaluation of the cumulated errors of the needle tracking, the patient model registration and the organ repositioning. It does not include the guidance error (defined in Sec. 2). Consequently, our experimental protocol allows us to evaluate all the error sources that only depend on the system and not on practitioner ability 1 . Alternatively, the measured error corresponds to the final system error if the needle insertion is robotized (in that case the guidance error is negligible).

${ }^{1}$ In fact, we measure a slight over-estimation of the system error: the needle registration from CT2 to CT1 is not perfect (we check its high accuracy in Sec. 4). 

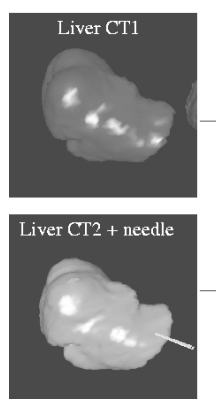
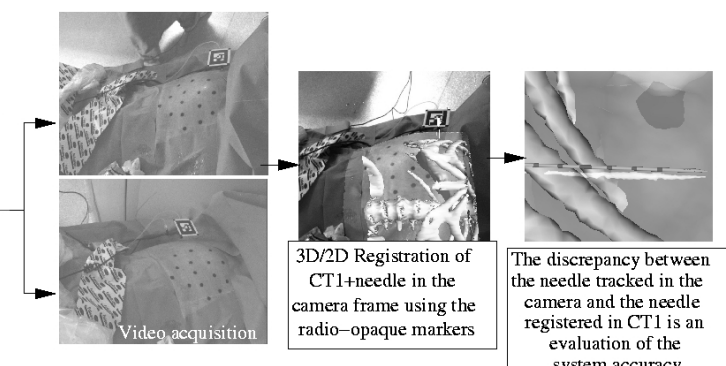

Fig. 4. Illustration of the passive protocol to evaluate the system accuracy

\section{Evaluation of the System on Six Clinical Cases}

Six patients ( 5 males and 1 female, age between 50 and 60) have participated in our experiments (they signed an agreement form). They all had tumors the diagnosis of which led to a RF thermal ablation. Resolution of CT images was $1 \times$ $1 \times 2 \mathrm{~mm}^{3}$. Below are presented the results obtained for the three experimental evaluations described in the previous section.

Verification of the needle rigidity assumption. One can see in Tab. 1 that the needle deflection is not negligible in $30 \%$ of cases as it can reach $2.5 \mathrm{~mm}$. Since the system assumes that the needle remains straight, the needle tip position provided by the system is systematically biased when there is an important deflection. Visual illustrations of a deflection are provided on Fig. 5 .

We are aware the practitioner sometimes bends the needle on purpose to avoid a critical structure. For all the reported cases, the practitioner estimated that this was not the case. Consequently, we have measured here the uncontrollable bending of the needle.

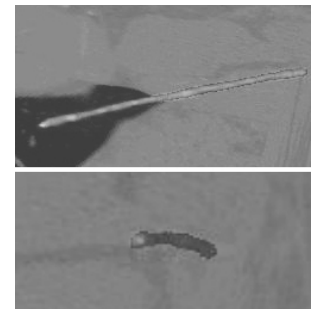

Fig. 5. Lateral and axial views of the needle (patient 2)

Table 1. Left table: Evaluation of the needle curvature after its positioning in the patient. Right table: Distance between the registered surfaces of spine, liver and skin.

\begin{tabular}{|l|c|c|}
\hline & $\begin{array}{c}\text { angular deviation } \\
\text { alpha }\left({ }^{\circ}\right)\end{array}$ & $\begin{array}{c}\text { needle } \\
\text { deflection }(\mathrm{mm})\end{array}$ \\
\hline \hline Patient 1 & 1.0 & 0.85 \\
\hline Patient 2 & 2.8 & 2.5 \\
\hline Patient 3 & 0.5 & 0.4 \\
\hline Patient 4 & 0.6 & 0.5 \\
\hline Patient 5 & 1.1 & 1.0 \\
\hline Patient 6 & 1.8 & 1.82 \\
\hline
\end{tabular}

\begin{tabular}{|c|c|c|c|}
\hline $\mathrm{d}\left(S_{1}, S_{2}\right)$ in $\mathrm{mm}$ & Spine & Liver & Skin \\
\hline \hline Patient 1 & 0.8 & 1.5 & 1.6 \\
\hline Patient 2 & 0.8 & 1.2 & 1.8 \\
\hline Patient 3 & 0.9 & 1.4 & 1.9 \\
\hline Patient 4 & 1.1 & 1.5 & 3.2 \\
\hline Patient 5 & 0.9 & 1.7 & 1.8 \\
\hline Patient 6 & 1.2 & 1.82 & 1.7 \\
\hline \hline Average & 0.95 & 1.6 & 2.0 \\
\hline
\end{tabular}


Evaluation of the organ repositioning error. To quantify the distance between two registered surfaces $S_{1}$ and $S_{2}$, we compute the average of the distance between each point on $S_{i}$ to the surface $S_{j}$ :

$$
d\left(S_{1}, S_{2}\right)=\frac{\sum_{M_{i} \in S_{1}} d\left(M_{i}, S_{2}\right)^{2}+\sum_{P_{i} \in S_{2}} d\left(P_{i}, S_{1}\right)^{2}}{2 \cdot\left(\operatorname{card}\left(S_{1}\right)+\operatorname{card}\left(S_{2}\right)\right)}
$$

where the distance $d\left(M_{i}, S\right)$ between a point $M_{i}$ and a surface $S$ is interpolated from the 3 closest points of $M_{i}$ belonging to $S$.

One can see in Tab. 1 (right) that the distance between liver surfaces is within $2 \mathrm{~mm}$ for each patient which is of the same magnitude as the segmentation uncertainty. To check that the measured distances are not due to a pure translation, we display the relative position of both surfaces. Fig. 6] (left columns) shows clearly that surfaces are closely interlaced for all patients. This means that the observed distance is essentially due to the segmentation error in the CT acquisitions and that the repositioning error of the liver is about $1 \mathrm{~mm}$.
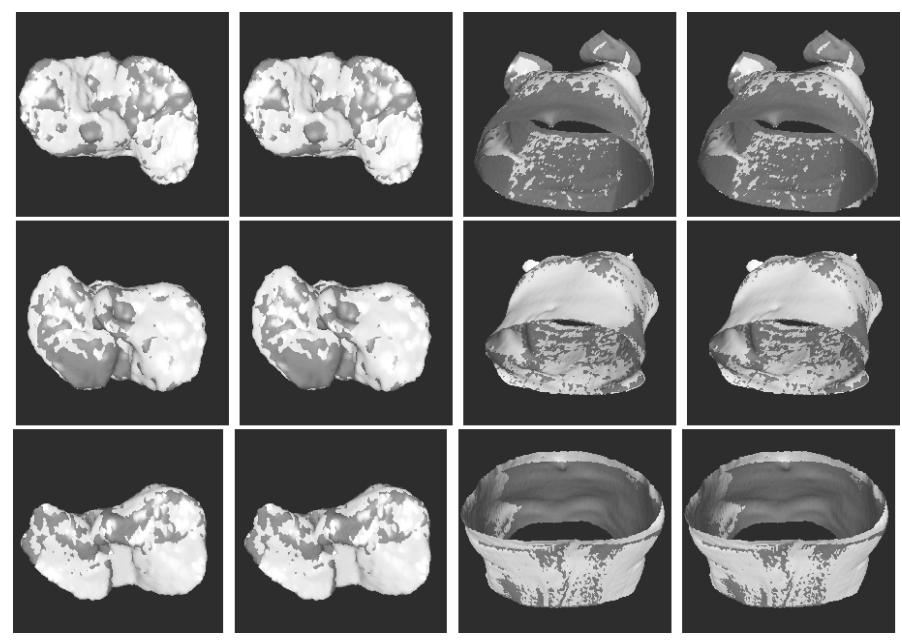

Fig. 6. Visual check of liver and skin repositioning errors on 3 patients (resp. left and right columns). Two opposite views of registered surfaces are provided for each patient.

Oddly, distances between skin surfaces are not very low for each patient. A visual check (see right columns in Fig. 6) of registered surfaces shows that for these patients the skin of the lower part of the abdomen has moved between the two CT acquisitions. An inspection of both CT indicates that a gas movement in the stomach and bowels was responsible for this deformation. We highlight that this skin deformation highly disturbs the system if we take the radio-opaque markers on the deformed zone into account to compute the patient model registration. Indeed, the system implicitly assumes that the relative position of the liver w.r.t. the markers remains rigid during the intervention. Consequently, the skin deformation can lead to a wrong estimation of the liver position. 
We notice that this phenomenon of gas movement essentially happened when the practitioner used the US probe. This means that the system should be carefully used if a US probe is manipulated. To avoid this problem, we can position the radio-opaque markers on the upper part of the abdomen only, which is a zone not influenced by gas movements. In the following we did not use radio-opaque markers on a deformed zone to evaluate the system accuracy.

Influence of breathing on the system accuracy. During the 6 interventions, the needle and the patient were video tracked along several breathing cycles. In Tab. 2, one can read for each patient the system accuracy, the $3 \mathrm{D} / 2 \mathrm{D}$ reprojection error of $\mathrm{CT}$ markers in the video images and the $3 \mathrm{D} / 3 \mathrm{D}$ registration error between CT markers and markers reconstructed from video images. These values were averaged on expiratory phases that were video recorded. Additionally, we report in Fig. 7 a sample for patients 1 and 2 during 4 breathing cycles of the system accuracy, the $3 \mathrm{D} / 2 \mathrm{D}$ and the $3 \mathrm{D} / 3 \mathrm{D}$ registration errors.
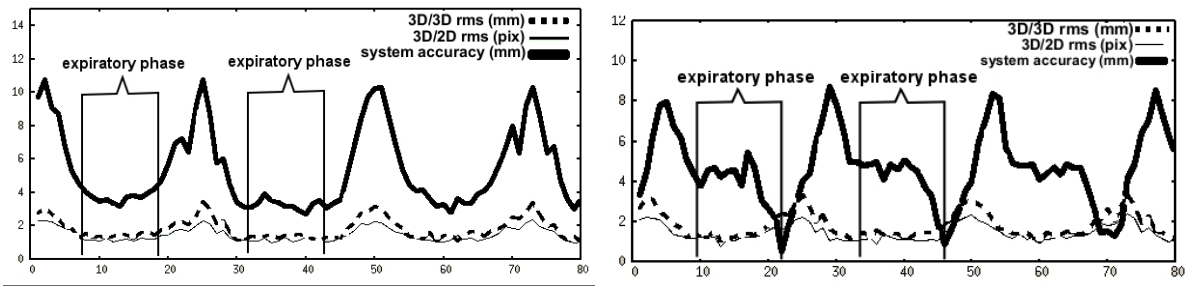

Fig. 7. Sample of system accuracy and registration errors reported during several breathing cycles with patients 1 and 2

Results in Tab. 2]indicate clearly that for all patients the system accuracy during expiratory phases is between 4 and $5 \mathrm{~mm}$. The two worst results have been obtained for patients whose abdominal zone has been deformed between the preoperative and control CT acquisitions. Indeed, in those cases, less markers could be used to compute the patient model registration. Note that including markers that had moved between the $\mathrm{CT}$ acquisitions in the registration computation leads to much worse accuracy (above $1 \mathrm{~cm}$ ).

For patients whose needle was bent, we have evaluated the system accuracy after having taken the observed curvature into account. This showed that if the rigidity assumption of the needle was true the system accuracy would be slightly better (about $0.5 \mathrm{~mm}$ ).

One can see in Fig 7 that RMS errors evolve cyclically, as expected, and are always minimal in expiration phases. The system accuracy also evolves cyclically but is not always minimal in expiration phases. Indeed, since the patient model registration is not perfect, the system can register the needle extracted from the $\mathrm{CT}$ at a position that corresponds to an intermediate phase of the breathing cycle (whereas it should be registered at expiratory position). 
Table 2. Average for each patient of the system error, $3 \mathrm{D} / 2 \mathrm{D}$ and $3 \mathrm{D} / 3 \mathrm{D}$ registration errors during expiration phases. The system provides an average guiding information during expiratory phases with an accuracy below $5 \mathrm{~mm}$. Values in brackets correspond to the results obtained when the markers on an abdominal zone deformed by gas motion are used for the patient model registration. Values in square brackets correspond to the system accuracy re-evaluated after a compensation of the important needle curvature (only for patients 2 and 6 ).

\begin{tabular}{|l|c|c|c|c|}
\hline & $\begin{array}{c}\text { Number of } \\
\text { markers used }\end{array}$ & $\begin{array}{c}\text { RMS 3D/2D } \\
\text { (pixel) }\end{array}$ & $\begin{array}{c}\text { RMS 3D/3D } \\
(\mathrm{mm})\end{array}$ & $\begin{array}{c}\text { System } \\
\text { accuracy }(\mathrm{mm})\end{array}$ \\
\hline \hline Patient 1 & 15 & 1.3 & 1.5 & 4.0 \\
\hline Patient 2 & 13 & 1.0 & 1.7 & $4.2[3.5]$ \\
\hline Patient 3 & $6(15)$ & $1.2(2.2)$ & $1.4(2.5)$ & $5.2(14.5)$ \\
\hline Patient 4 & 12 & 1.5 & 1.2 & 4.1 \\
\hline Patient 5 & $8(13)$ & $0.9(2.0)$ & $1.5(2.4)$ & $4.8(12.3)$ \\
\hline Patient 6 & 14 & 1.2 & 1.2 & $4.3[3.9]$ \\
\hline \hline Average & 11.5 & 1.18 & 1.44 & $\mathbf{4 . 3}$ \\
\hline
\end{tabular}

\section{Conclusion}

We have developed a computer system to guide liver percutaneous punctures in interventional radiology. To tackle the breathing motion issue that induces a movement of the liver (above $1 \mathrm{~cm}$ ), we propose to use a respiratory gating technique. We synchronize the preoperative CT acquisition and the guidance step with the expiratory phases of the patient. Then, we assume pseudo static conditions and rigidly register the patient model.

To assess rigorously the system accuracy and the pseudo static assumption on real patients, we propose a passive protocol on carefully chosen patients that allows us to obtain a ground truth CT at the end of the needle insertion. Experimental results show firstly that the liver repositioning error is about 1 $\mathrm{mm}$ whereas it is sometimes much more important for the skin because of gas movement in the bowels. This phenomenon can dramatically decrease the system accuracy if markers on the deformed zone are used to compute the patient model registration. Therefore, to avoid this problem, markers have to be positioned only around the ribs. Secondly, we have evaluated that the needle curvature can cause a needle tracking error above $2 \mathrm{~mm}$ (although the practitioner thought it was not bent). Despite these uncertainties, we have finally showed that our system accuracy during the patient expiratory phases is about $4.5 \mathrm{~mm}$, which fits the medical requirements.

We investigate now the integration of an electromagnetic tracker in the current system so that we will be able to directly track the needle tip (although this is still a challenge due to ferromagnetic object presence in the operating room). Last but not least, a validation step including the needle manipulation by the practitioner is planned for next year. 


\section{References}

1. Balter, J.M., et al.: Improvement of CT-based treatment-planning models of abdominals targets using static exhale imaging. IJROBP 41(4), 939-943 (1998)

2. Dawson, L., et al.: The reproducibility of organ position using active breathing control (ABC) during liver radiotherap. IJROBP 51, 1410-1421 (2001)

3. Fichtinger, G., et al.: Image overlay guidance for needle insertion in ct scanner. IEEE Transaction on Biomedical Engineering 52(8), 1415-1424 (2005)

4. Hostettler, A., Nicolau, S.A., Forest, C., Soler, L., Remond, Y.: Real time simulation of organ motions induced by breathing: First evaluation on patient data. In: Harders, M., Székely, G. (eds.) ISBMS 2006. LNCS, vol. 4072, pp. 9-18. Springer, Heidelberg (2006)

5. Mitschke, M., et al.: Interventions under video-augmented X-ray guidance: Application to needle placement. In: Delp, S.L., DiGoia, A.M., Jaramaz, B. (eds.) MICCAI 2000. LNCS, vol. 1935, pp. 858-868. Springer, Heidelberg (2000)

6. Nicolau, S., et al.: An augmented reality system to guide radio-frequency tumor ablation. Jour. of Computer Animation and Virtual World 16(1), 1-10 (2005)

7. Nicolau, S., Pennec, X., Soler, L., Ayache, N.: An accuracy certified augmented reality system for therapy guidance. In: Pajdla, T., Matas, J(G.) (eds.) ECCV 2004. LNCS, vol. 3023, pp. 79-91. Springer, Heidelberg (2004)

8. Nicolau, S., Schmid, J., Pennec, X., Soler, L., Ayache, N.: An augmented reality \& virtuality interface for a puncture guidance system: Design and validation on an abdominal phantom. In: Yang, G.-Z., Jiang, T. (eds.) MIAR 2004. LNCS, vol. 3150, pp. 302-310. Springer, Heidelberg (2004)

9. Nicolau, S.A., Pennec, X., Soler, L., Ayache, N.: A complete augmented reality guidance system for liver punctures: First clinical evaluation. In: Duncan, J.S., Gerig, G. (eds.) MICCAI 2005. LNCS, vol. 3749, pp. 539-547. Springer, Heidelberg (2005)

10. Patriciu, A., et al.: Robotic assisted radio-frequency ablation of liver tumors:a randomized patient study. In: Duncan, J.S., Gerig, G. (eds.) MICCAI 2005. LNCS, vol. 3750, pp. 526-533. Springer, Heidelberg (2005)

11. Pereira, P.L.: Actual role of radiofrequency ablation of liver metastase. In: European Radiolology (February 15, 2007)

12. Remouchamps, V., et al.: Significant reductions in heart and lung doses using deep inspiration breath hold with active breathing control and intensity-modulated radiation therapy for patients treated with locoregional breast irradiation. IJROBP 55, 392-406 (2003)

13. Soler, L., et al.: Fully automatic anatomical, pathological, and functional segmentation from CT scans for hepatic surgery. Computer Aided Surgery 6(3) (2001)

14. Wacker, F., et al.: An augmented reality system for MR image-guided needle biopsy: Initial results in a swine model. Radiology 238(2), 497-504 (2006)

15. Wagman, R., et al.: Respiratory gating for liver tumors: use in dose escalation. Int. J. Radiation Oncology Biol. Phys. 55(3), 659-668 (2003)

16. Wong, J., et al.: The use of active breathing control (ABC) to reduce margin for breathing motion. Int. J. Radiation Oncology Biol. Phys. 44(4), 911-919 (1999) 